\title{
Design of ADS-B Simulator
}

\author{
Hyeon-Cheol Lee, Sang Gyu Lee, and Sang Soon Yong \\ Satellite Payload Development Division, Satellite R\&D Head Office, Korea Aerospace Research Institute, \\ 169-84, Gwahak-ro, Yuseong-gu, Daejeon, 34133, Rep. of Korea
}

\begin{abstract}
In this paper, the ADS-B (automatic dependent surveillance-broadcast) used for manned aircrafts is chosen for a See-and-Avoid sensor of UAV (unmanned aerial vehicle), then GDL90 type is selected among three types of ADS-B. The serial communication data protocol which communicates between GDL90 and control panel of GDL90 is analyzed and tailored for the interface of the GDL90 simulator here. This ADS-B (GDL90) simulator sends intruder information to FCC (flight control computer) of UAV for collision avoidance function.
\end{abstract}

Key words: ADS-B, GDL90, 1090ES, UAV, collision-avoidance.

\section{Introduction}

The UAV (unmanned air vehicle) is widely developed in the world recently, one of the important functions of UAV Program is a collision avoidance system which generates new detour path for avoiding flying intruders or manned aircrafts, when UAVs go into the manned airspace.

The technologies of the collision avoidance system are differentiated into two parts, a See-and-Avoid sensor and design of collision avoidance algorithm. The See-and-Avoid sensor is further divided into two parts again, active sensor and passive sensor. It is passive, if opposite intruders provide necessary information for our own sensor, but, it is active, if not. The active one, however costs a lot for implementing the system, therefore the passive one is preferred for general usage. The passive sensors are TCAS (traffic alert and collision avoidance system) or ADS-B (automatic dependent surveillance-broadcast), the active sensors are RADAR or Video detector, etc.

These sensors have been used for manned aircrafts, we, however introduce here how to use these sensors for unmanned aircraft to avoid collisions in the manned airspace. The ADS-B, instead of TCAS is chosen for

Corresponding author: Hyeon-Cheol Lee, Ph.D., principal researcher, research fields: communication and SAR. the collision avoidance system for UAV program in this paper since the communication range is three times longer and more specific data exchange between aircraft and ground controller, or between aircraft and aircraft. The types $[1,2]$ of ADS-B are in Table 1.

GDL90, the UAT (universal access transceiver) type widely used in North America among three types of ADS-B is chosen for UAV program usually because 1090ES has large bandwidth consumption and lack of bidirectional capability. GDL90 and display of MFD (multi functional display) are shown in Fig. 1 and Fig. 2 , respectively.

In this paper, serial communication data protocol [3] between GDL90 and control panel of GDL90 is analyzed for UAV program, then this protocol is tailored to the design of a GDL90 simulator that interacts with an FCC (flight control computer) capable of controlling UAV and receiving data from UAV. GDL90 can send up to 32 numbers of intruder information and its own vehicle information to FCC and this FCC can verify its collision avoidance algorithm with the GDL90 simulator.

This paper is organized as follows. Message structure of the serial data protocol between GDL90 and control panel of GDL90 is described in Section 2. Tailored serial data protocol is introduced in Section 3. Operational approach of GDL90 for UAV program is 
Table 1 Types of ADS-B.

\begin{tabular}{lll}
\hline ADS-B & Area & Frequency \\
\hline VDL mode 4 & Europe & VHF \\
1090ES & World & $1,090 \mathrm{MHz}$ \\
UAT & North America & $978 \mathrm{MHz}$ \\
\hline
\end{tabular}

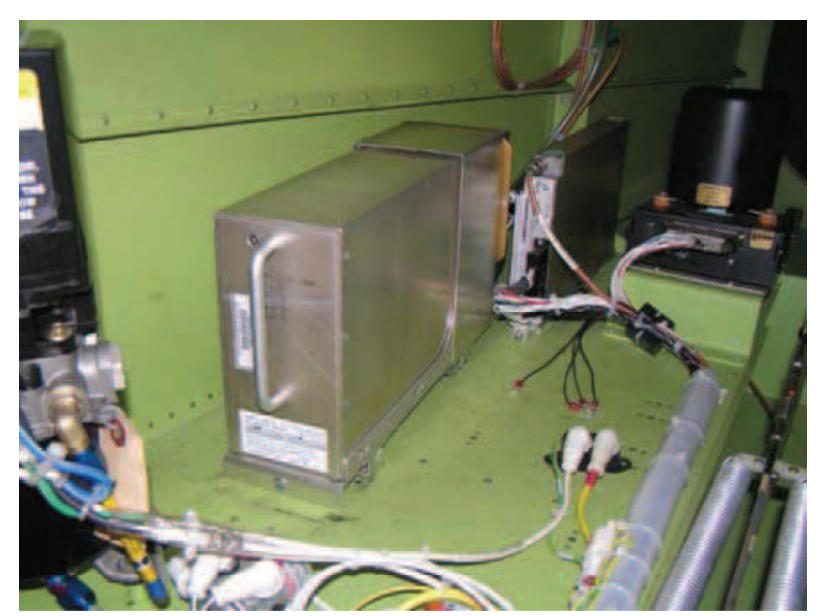

Fig. 1 GDL90.

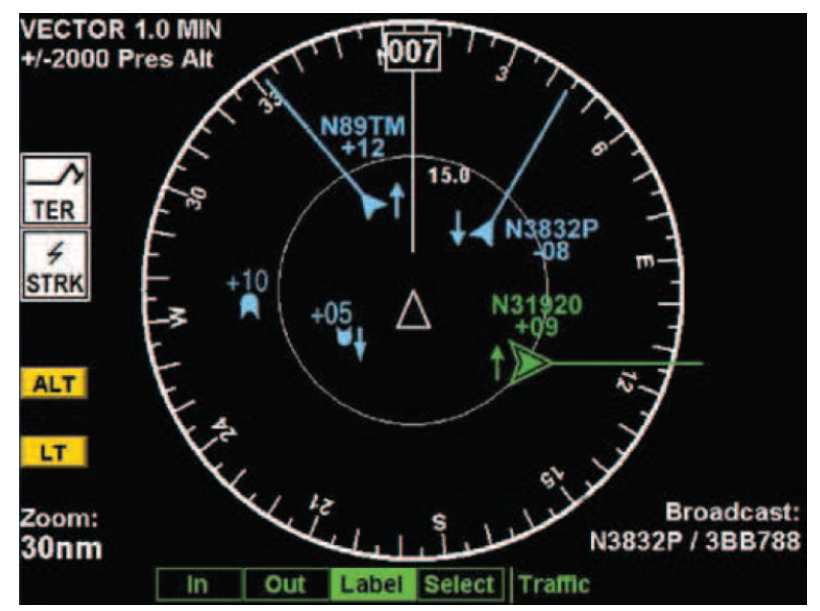

Fig. 2 MFD of GDL90.

shown in Section 4. The GDL90 simulator is introduced in Section 5. Finally, conclusion is drawn in Section 6.

\section{Introduction of GDL90}

Fig. 3 shows an airborne interface diagram of GDL90 when it is installed on a manned aircraft. Airborne equipment consists of GDL90, control panel, altitude encoder [4], and MFD for interaction with a pilot. GDL90 transmits or receives information to/from a control panel by RS-422 serial data protocol.

FCC in UAV needs to connect GDL90 instead of control panel and this FCC communicates a pilot in GCS (ground control station) by up/downlink of wireless communication. The altitude encoder (pressure type altimeter) sends altitude information of its own vehicle to GDL90. MFD shows all information to a pilot on a manned vehicle. This RS-422 serial data protocol is a bi-directional asynchronous interface, 38,400 bps, 1 (start bit), 1 (stop bit), N (parity bit). Maximum speed is up to 3,500 byte/sec when it considers $10 \%$ margin for tracking acquisition and datalink interface. This serial data protocol follows below message structure and type and size of the messages are shown in Table 2.

Message Structure:

Flag(1byte : $0 \times 70)+\operatorname{msgID}(1$ byte, Table 2$)+$ msgdata(Table 2$)+\mathrm{CR}(2$ byte $)+$ flag $(1$ byte $: 0 \times 70)$

The initialization is an input signal to GDL90 for initializing GDL90. Height Above Terrain is altitude input data from the altitude encoder connected to GDL90 externally. The other signals are outputs from

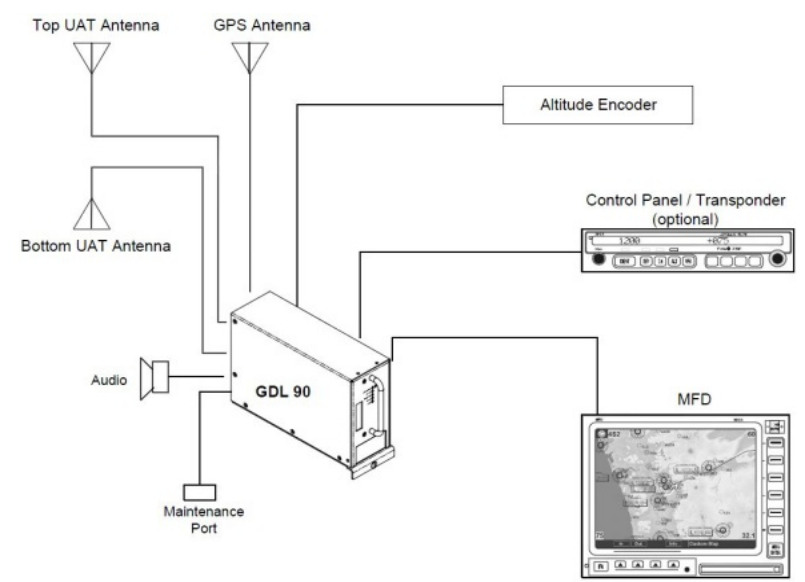

Fig. 3 Interface diagram of GDL90 on a manned aircraft.

Table 2 Type and size of the messages of GDL90 (msgID size is excluded).

\begin{tabular}{llll}
\hline Message & msgID & Byte size & I/O \\
\hline Heartbeat $(1 \mathrm{~Hz})$ & $0 \mathrm{~d} 00$ & 6 & Out \\
Uplink (4 Hz) & $0 \mathrm{~d} 07$ & 435 & Out \\
Ownship Report $(1 \mathrm{~Hz})$ & $0 \mathrm{~d} 10$ & 27 & Out \\
Traffic Report $(1 \mathrm{~Hz})$ & $0 \mathrm{~d} 20$ & $32 * 27$ & Out \\
Basic Report & $0 \mathrm{~d} 30$ & 21 & Out \\
Long Report & $0 \mathrm{~d} 31$ & 37 & Out \\
Initialization $(1 \mathrm{~Hz})$ & $0 \mathrm{~d} 02$ & 2 & Out \\
Height Above Trn & $0 \mathrm{~d} 09$ & 2 & Out \\
\hline
\end{tabular}


GDL90, such as Heartbeat, Uplink Data, Ownship Report, Traffic Report, Basic and Long Report. The Uplink Data shows that the aircraft information including manned and unmanned vehicles gathered by a ground equipment, GBT (ground-based transceiver) (manufactured by Sensis Corporation) [5] is sent from GBT by $978 \mathrm{MHz}$ to airborne GDL90. This protocol including Heartbeat, Ownship, Traffic Report was tailored for UAV program, then described in Section 3. Initialization and Height Above Terrain, however is not used for the GDL90 simulator. NIC (navigation integrity category) and NACp (navigation accuracy category for position) not mentioned here mean integrity and accuracy, respectively.

\section{Serial Communication Data Protocol}

\subsection{Heartbeat}

Heartbeat produces a synchronization signal for every second according to the UTC timing reference signal of GDL90.

\subsection{Ownship Report}

Ownship Report produces location of its own vehicle and the format is same as Traffic Report described in next section.

\subsection{Traffic Report}

Traffic Report of intruders shown in Table 3 can produce maximum 32 number of vehicle information, therefore, the data size is $32 * 28$ bytes. In this paper, traffic alert, latitude, longitude, altitude, horizontal velocity, vertical velocity, and tracking/heading are described.

\subsubsection{Traffic Alert}

No alert if it is $0 \times 00$, traffic alert if it is $0 \times 01$. Once traffic alert is on, GDL90 starts to detect intruders.

\subsubsection{Latitude, Longitude}

Longitude and latitude shown in Table 4 represent 24-bit semicircle 2-s complement units. These are encoded in range of $+/-180$ degrees with resolution of $2.14577 \mathrm{E}-5$ (approximately 2.38 meters at the equator).
For latitude, East is positive, for longitude, North is positive. Maximum/minimum latitude value is $+/-90.0$ degrees.

\subsubsection{Altitude}

Altitude shown in Table 5 is encoded using 25-foot resolution, offset by $1,000 \mathrm{ft}$, therefore, the minimum altitude is $-1,000 \mathrm{ft}(0 \times 000)$ and maximum is 101,350 $\mathrm{ft}(0 \times \mathrm{FFE})$. Altitude is invalid if it is $0 \times \mathrm{FFF}$.

\subsubsection{Horizontal Velocity}

This horizontal velocity is encoded as 12-bit unsigned value with resolution of 1 FPM (feet per minute). Direction is in the tracking/heading field. Data

Table 3 Format of traffic report.

\begin{tabular}{llll}
\hline Byte & Bit & Items & Remark \\
\hline 0 & $7-4$ & Traffic alert & \\
$1-3$ & $3-0$ & Address type & \\
$4-6$ & $7-0$ & Participant addr. & \\
$7-9$ & $7-0$ & Latitude & \\
10 & $7-0$ & Longitude & \\
11 & $7-0$ & Altitude & \\
11 & $7-4$ & & Integrity \\
12 & $3-0$ & NIC & Accuracy \\
13 & $7-4$ & NACq & \\
14 & $7-0$ & Horizontal velocity & \\
14 & $7-4$ & & \\
15 & $7-0$ & Vertical velocity & \\
16 & $7-0$ & & \\
17 & $7-0$ & Track/heading & \\
$18-25$ & $7-0$ & Call sign & ASCII \\
26 & $7-4$ & Emergency code & \\
& $3-0$ & Spare & \\
\hline
\end{tabular}

Table 4 Latitude, longitude.

\begin{tabular}{ll}
\hline Degree & Hex \\
\hline $45.0 \mathrm{~N}$ or $\mathrm{E}$ & $0 \times 200000$ \\
$180.0 \mathrm{~N}$ or $\mathrm{E}$ & $0 \times 7 \mathrm{FFFFF}$ \\
$45.0 \mathrm{~S}$ or W & $0 \times \mathrm{E} 00000$ \\
$180.0 \mathrm{~S}$ or $\mathrm{W}$ & $0 \times 800000$ \\
\hline
\end{tabular}

Table 5 Altitude.

\begin{tabular}{lll}
\hline Altitude & Hex & Altitude calculation \\
\hline$-1,000 \mathrm{ft}$ & $0 \times 000$ & $0 \mathrm{~d} 00 * 25-1000$ \\
$+0.0 \mathrm{ft}$ & $0 \times 028$ & $0 \mathrm{~d} 40 * 25-1000$ \\
$+1,000 \mathrm{ft}$ & $0 \times 050$ & $0 \mathrm{~d} 80 * 25-1000$ \\
$+101,350 \mathrm{ft}$ & $0 \times \mathrm{FFE}$ & $0 \mathrm{~d} 4094 * 25-1000$ \\
\hline
\end{tabular}


range is $0 \mathrm{ft}(0 \times 000)-4,094 \mathrm{ft}(0 \times \mathrm{FFE})$. It is invalid if data show $0 \times \mathrm{FFF}$.

\subsubsection{Vertical Velocity}

This vertical velocity shown in Table 6 is encoded as 12-bit signed value with resolution of 64 FPM. The data use 2-s complement units for descend value. Data range is $-32,576 \mathrm{FPM}(0 \times \mathrm{E} 03)-+32,576 \mathrm{FPM}$ $(0 \times 1 \mathrm{FD})$, and it is $0 \times 1 \mathrm{FE}$ if data is over $+32,576 \mathrm{FPM}$ climb, it is $0 \times \mathrm{E} 02$, if data is over $+32,576 \mathrm{FPM}$ descend.

\subsubsection{Track/Heading}

Heading is shown in Table 7, it is North direction if hexa value is $0 \times 00$, it is South direction if hexa value is $0 \times 80$. Its resolution is $1.40625 \mathrm{deg}$.

\subsubsection{Emitter Category}

This emitter category with range 0 to 39 is classified by vehicle size and vehicle class.

\section{Operational Approach for UAV}

Fig. 4 shows operational approach of GDL90 as a See-and-Avoid sensor for UAV program. After traffic alert is initiated while FCC is watching traffic alert, FCC tries to identify an intruder through address type registered in ICAO. After that, GDL90 locates longitude, latitude, and altitude of the intruder information, then computes moving direction of the intruder with horizontal velocity, vertical velocity, and heading. If other intruders are found, GDL90 starts same procedure up to 32 numbers of intruders. Therefore, the collision avoidance algorithm of FCC starts to initiate.

\section{Simulation Design}

Based on the tailored serial data protocol, the GDL90 simulator for interfacing FCC is designed. Input data, the Initialization and Height Above Terrain are not necessary for the simulator. This simulator works same as GDL90 itself, but it can produce and send maximum 32 numbers of intruder information to FCC for verifying the collision avoidance algorithm of FCC since GDL90 cannot produce intruders unless real intruders appear.

Table 6 Vertical velocity.

\begin{tabular}{ll}
\hline Vertical velocity & Hex. \\
\hline 64 FPM climb & $0 \times 0001$ \\
32,576 FPM climb & $0 \times 1 \mathrm{FD}$ \\
64 FPM descend & $0 \times \mathrm{FFF}$ \\
32,576 FPM descend & $0 \times \mathrm{E} 03$ \\
\hline
\end{tabular}

Table 7 Heading.

\begin{tabular}{lll}
\hline Heading & Hex & Deg. calculation \\
\hline $000.0 \mathrm{deg}$ (North) & $0 \times 00$ & $0 \mathrm{~d} 000 * 1.40625$ \\
$090.0 \mathrm{deg}$ (East) & $0 \times 40$ & $0 \mathrm{~d} 064 * 1.40625$ \\
$180.0 \mathrm{deg}$ (South) & $0 \times 80$ & $0 \mathrm{~d} 128 * 1.40625$ \\
$270.0 \mathrm{deg}$ (West) & $0 \times \mathrm{C} 0$ & $0 \mathrm{~d} 192 * 1.40625$ \\
\hline
\end{tabular}

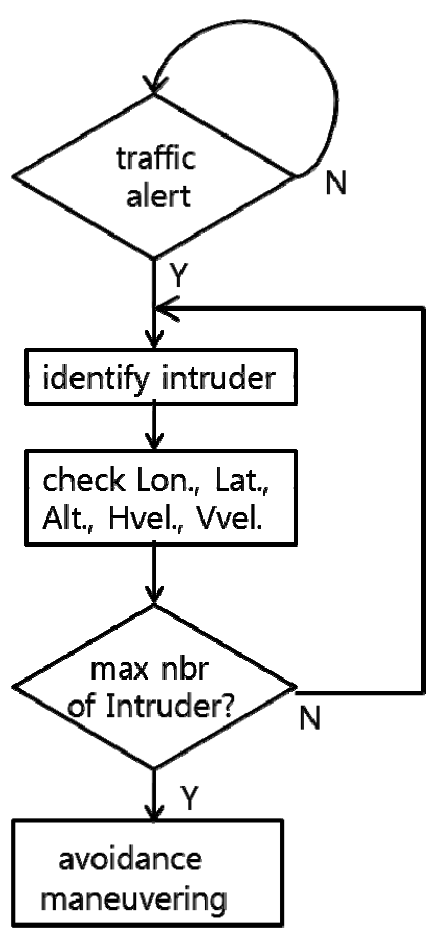

Fig. 4 Flowchart of operational approach for UAV.

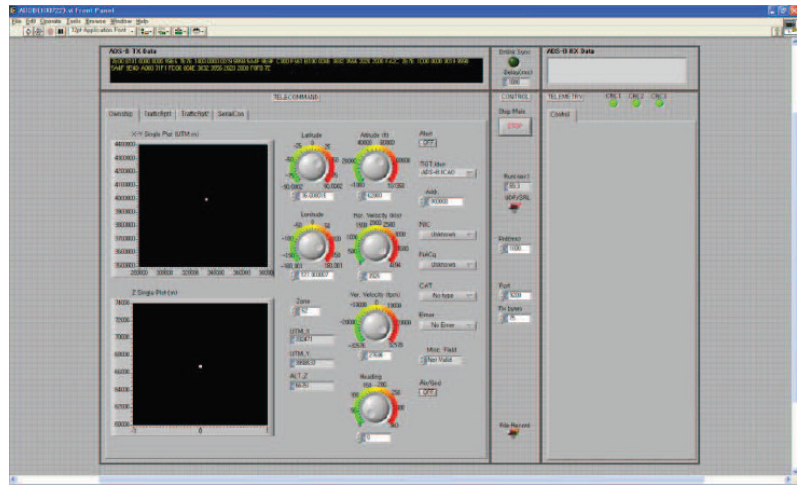

Fig. 5 GDL90 simulator. 
Fig. 5 shows the simulator designed by LabView. The GDL90 simulator starting from initial location of intruders with given latitude, longitude, and altitude shows the moving trajectories of intruders by calculating horizontal velocity and heading values in $\mathrm{X}-\mathrm{Y}$ plot display, the varied altitude of intruders by calculating vertical velocity value in $\mathrm{Z}$ plot display. Other options, such as address type, identification number, NIC, NACq, category, call sign, emergency code are sent together with intruder information. With the intruder information given to FCC, FCC operates its collision avoidance algorithm.

\section{Conclusion}

GDL90, the UAT type of ADS-B among passive See-and-Avoid sensors was chosen for the UAV collision avoidance system. The communication data protocol between GDL90 and control panel of GDL90 was tailored for UAV collision avoidance algorithm. Then, an operator in GCS can operate See-and-Avoid sensor using the airborne GDL90 through FCC by up/downlink of UAV datalink.

In this paper, ADS-B simulator using GDL90 was designed based on the tailored serial data protocol between GDL90 and its control panel. FCC can verify its own collision avoidance algorithm with the intruder signal sent by the ADS-B simulator.

\section{References}

[1] ADS-B 1090ES Extraction User Manual, Oct. 2010.

[2] GDL90 Data Interface Specification, Aug. 2004.

[3] Altitude Encoder/Digitizer Owner/Installation Manual, Aug. 2009.

[4] Ground-Based Transceiver (GBT) for Broadcast Services Using the Universal Access Transceiver (UAT) Data Link, Jan. 2004. 